\title{
New Synthesis - Visual and Chemical Ornaments: What Researchers of Different Signal Modalities Can Learn from Each Other
}

\author{
Sandra Steiger \\ Published online: 14 January 2012 \\ (C) Springer Science+Business Media, LLC 2012
}

Interestingly, the field of animal communication appears to suffer from a lack of communication between scientists studying different sensory channels. Communication theory has been developed primarily on the basis of visual and acoustic signals; chemical signals have been neglected, despite a long history of outstanding studies and a plethora of available literature. Conversely, chemical ecologists tend to focus on proximate mechanisms, often ignoring the concept structure developed by behavioral and evolutionary ecologists. Clearly, researchers of chemical and non-chemical communication would benefit from each other's ideas, and a joint effort could generate new insight into why and how signals evolve. A recent article by Hill (2011) proposes a useful framework to this end.

Condition and condition-dependent traits are well-known terms in the community working on communication and sexual selection, but as Hill (2011) points out they are poorly defined. He defines condition as the capacity to maintain system functionality and to withstand environmental challenges, and condition-dependent traits as "conspicuous features of an organism that enhance perception of condition". Moreover, he proposes four distinct if nonexclusive ways that ornaments (although he means visual ornaments, we can expand it to include chemical ornaments) can be linked to system functionality: 1) Resource Tradeoff Hypothesis: resources needed for signal production also are needed for the operation of essential physiological processes; 2) Mediator Hypothesis: a regulatory agent (e.g., hormone) promotes signal production, but suppresses a vital physiological process; 3) Pathway Functionality Hypothesis: signal production requires a product of a vital physiological process; and 4) Shared Pathway Hypothesis: signal production and vital systems share pathways. These hypotheses are not entirely new to pheromone researchers, as they invoke the biosynthesis of such chemical signals. However, the different possibilities of linkage between condition and the signal have never been stated as explicitly as Hill has done.

Most available evidence seems to support the first hypothesis, as pheromone synthesis often depends on limited nutritional resources. Yet, few studies provide a clear demonstration of a linkage between the specific resource and essential physiological processes. Exceptions are the well known examples of male moths and butterflies that sequester alkaloids derived from food plants. The alkaloids are essential for their own defense and the defense of their offspring, but also are the resource for sex pheromone production. A more recent exciting example is the study of Blaul and Ruther (2011), which demonstrates that dietary linoleic acid is not only a precursor of the male sex pheromone in Nasonia vitripennis wasps, but also affects sperm production and therefore male condition.

Evidence supporting Hill's second hypothesis comes from work that shows that juvenile hormone $(\mathrm{JH})$ promotes pheromone production but suppresses immunity. However, the exact mechanism of this linkage is unknown,

S. Steiger $(\bowtie)$

Universität Regensburg, Regensburg, Germany

e-mail: Sandra.Steiger@biologie.uni-regensburg.de and as Hill (2011) pointed out for testosterone, JH might only regulate energy allocation, shunting either the energy to general homeostasis or to the production of pheromones. In this case, the Mediator Hypothesis would not differ from the Resource Tradeoff Hypothesis.

Hill's third and fourth hypotheses both predict that the pathway of ornament production is intimately linked to some essential physiological pathways, and thus are likely relevant to chemical signals. Evidence comes from studies on lizards (Lopez et al. 2009). Here, sex pheromone and Vitamin D production share the same biosynthetic pathway; more precisely, the pheromone is a precursor of Vitamin D. In mammals, Vitamin D positively influences immunity, and the same might be true for lizards (see references in Lopez et al. 2009). Another example comes from insects, in which many pheromones share their pathways with fatty acid metabolism, a critical physiological process responsible for supplying energy to the body. A high rate of pheromone production is possible only if lipid metabolism is functioning well. However, here we can also see the limitation of Hill's approach. Nearly each insect pheromone synthesized de novo is tightly linked to a pathway that is important for maintenance of system functionality. Does that mean that all these pheromones are condition dependent signals and used in mate choice? Presumably not. They only reflect condition if (1) pheromone production reaches a critical level, a level that reflects metabolic efficiency, otherwise there would not be much variation in chemical signaling between individuals. (2) The shared essential physiological pathway has to be prone to disturbance or somehow depend on individual differences in phenotype, otherwise there would be not much variability in condition and hence signal quantity or quality between individuals. I encourage chemical ecologists interested in sexual selection to focus on these last two mentioned aspects and investigate whether or not the metabolic machinery places any strain on signal production. In recent decades, the deciphering of metabolic pathways that connect visual signal production to condition has been a neglected research area. In contrast, the specific pathways of chemical signal production have been investigated extensively and, therefore, chemical ecologists are well positioned to provide new insights into the proximate mechanisms of sexually selected signals, from which researchers of all signal modalities would benefit.

\section{References}

Blaul, B., and Ruther, J. 2011. How parasitoid females produce sexy sons: a causal link between oviposition preference, dietary lipids and mate choice in Nasonia. Proc. R. Soc. B 278: 32863293.

HILL, G. E. 2011. Condition-dependent traits as signals of the functionality of vital cellular processes. Ecol. Lett. 14:625-634.

LoPeZ, P., GABIROT, M., and MARTIN, J. 2009. Immune activation affects chemical sexual ornaments of male Iberian wall lizards. Naturwissenschaften 96:65-69. 